\title{
Managing Stimulation of Regional Innovation Subjects' Interaction in the Digital Economy
}

\author{
Submitted 23/06/19, 1st revision 11/07/19, 2nd revision 25/09/10, accepted 20/10/19
}

\section{Sergey Vasin ${ }^{1}$, Leyla Gamidullaeva ${ }^{2}$, Alexey Finogeev ${ }^{3}$}

\begin{abstract}
:
Purpose: The article is devoted to solving fundamental scientific problems in the scope of the development of forecasting modeling methods and evaluation of regional company's innovative development parameters, synthesizing new methods of big data processing and intelligent analysis, as well as methods of knowledge eliciting and forecasting the dynamics of regional innovation developments through benchmarking.

Design/Methodology/Approach: For regional economic development, it is required to identify the mechanisms that contribute to (or impede) the innovative economic development of the regions. The synergetic approach to management is based on the fact that there are multiple paths of IS development (scenarios with different probabilities), although it is necessary to reach the required attractor by meeting the management goals.

Findings: The present research is focused on obtainment of new knowledge in creating a technique of multi-agent search, collection and processing of data on company's innovative development indicators, models and methods of intelligent analysis of the collected data.

Practical Implications: The author developed recommendations before starting the process of institutional changes in a specific regional innovation system. The article formulates recommendations on the implementation of institutional changes in the region taking into account the sociocultural characteristics of the region's population.

Originality/Value: It is the first time, when a complex of models and methods is based on the use of a convergent model of large data volumes processing is presented.
\end{abstract}

Keywords: Industry 4.0, manufacturing, industrialization, digital economy.

JEL Codes: G21, G29.

Article type: Research article.

Acknowledgement:

The reported study was funded by RFBR according to the research project No. 18-01000204_a,No.16-07-00031_a,No.18-07-00975_a.

\footnotetext{
${ }^{1}$ Penza State University, Russia

${ }^{2}$ Penza State University, Russia, gamidullaeva@gmail.com

${ }^{3}$ Penza State University, Russia
} 


\section{Introduction}

Elements of the innovation environment of regional innovation systems are dissociated due to the insufficient interaction between them. In our opinion, the main obstacle impeding efficient interaction of innovation subjects in Russia is high transaction costs. Here, it is necessary to simulate interaction of main innovation participants in a regional innovation system to reach qualitative and quantitative understanding of the significance of links between the innovation environment's elements, the effect of their energy interaction on attractors, on energy potentials of system's order parameters.

The development of interaction between all interested participants in an innovation system is a crucial mechanism of innovation activity development at various stages of the innovation process, from the origin of an idea to the commercialization of innovations. It should be taken into account that the intermediate stages of the innovation activity feature growing instability and uncertainty of innovation company's existing connections and relations, thus causing a growth of transaction costs. It determines a high level of innovation expenditures being mostly not of transformational (associated with conversion and changing of initial resources), but of transactional (mostly caused by a necessity to collaborate and establish contacts) nature. Consequently, we believe, the main barrier encountered by the innovative development of socioeconomic systems is transactional costs that demotivate business-owners to implement innovations.

The process of innovation creation is a result of close collaboration between various participants of an innovation system that depends on transactions within a company, as well as on existing relations of an economic agent with the institutional medium. The institutional medium, in turn, establishes conditions to develop interaction of economic agents with other participants of an innovation system (IS) regarding the search for information, experience and other resources, influences and even determines the behavior of innovation economic agents by shaping their behavioral sets, formal institutional structures crucial when it comes to decision-making. According to the chosen methodological approach, the regional innovation system is a system of interaction between economic agents, as well as a system of formal and informal institutions of a regional innovation environment.

We should emphasize that innovation process participants should collaborate without hindrance, accumulate experience from completing joint projects intended to create and commercialize innovations. This, in turn, requires efficient coordinated relations within the "subject-project-medium" continuum. The key process in stimulating interaction of a subject, a project and a medium is self-organization (improving the organization and order of interaction) providing a decrease of transaction costs and stimulating a growth of the joint effect. Participants of the innovation activity cannot obtain these benefits separately. It should be specified that under transaction costs reduction we understand not a decrease to zero, but 
optimization within normal values, as such costs are objectively needed for economic system functioning. Therefore, innovation system management should aim at development and realization of the corresponding strategy ensuring identification of attracting structures that most precisely correspond to main paths of development and take into account the overall situation and participants' capabilities.

To form and support an innovation system it is expedient to use a cyber-social system that makes the interaction of innovation activity participants simpler and stimulates their collaboration through analyzing a current situation (available data on a project) and selecting solutions to occurring problems. The cyber-social system would be one of innovation system's participants and by interacting with other participants it would provide the synergetic effect by virtue of cooperative contribution by all participants. Essentially the cyber-social system is an adaptive intelligent information system merging social and intelligent processes designed in the form of an Internet-portal that all participants of a regional innovation system (objects of an innovation infrastructure; universities and research centers; small, medium and large companies; state bodies and structures; users and the community) should be connected to.

Consequently, the regional innovation system may be considered as an intelligent cyber-social system focused on lowering of innovation implementation barriers (high transaction costs of the interaction of innovation activity subjects) by attracting more participants to the innovation process and securing their intensive and efficient collaboration.

Thus, it is the first time when innovation system management is suggested to be realized on the basis of the cyber-social intelligent system with elements of artificial intelligence capable of organizing interaction of users and adapting to their needs, analyzing current situations and elaborating expedient solutions, out of which users can select best ones to be implemented.

\section{Literature Review}

To research the problem of stimulating the interaction of innovation economy's subjects it is necessary to consider a number of basic concepts outlining the theory and methodology of the present research. Due to the complexity of products and the interdisciplinary nature of technologies applied, innovations more and more depend on external knowledge remaining outside certain companies. In recent years, some developed countries have found a use in innovation activities for the conception of open innovations (Chesbrough, 2003) that considers research and development as an open dynamic system. Consequently, sources of new knowledge may be located both inside and outside a company. In the second case, continuous high-speed information exchange may result in competitive advantage in local markets provided rapid implementation of new technologies created outside a company. Even powerful international companies strive to combine internal networks of their sub- 
divisions involved in generation, storage, usage and absorption of knowledge with external networks including companies, universities, institutions associated with the company's sub-divisions. It amplifies the internal potential of generation, absorption and application of new knowledge. At the same time, there is more and more evidence of the fact that the adoption of the open innovations conception accelerates research and development, as well as makes it profitable and diverse. As a result, this conception is compatible with corporative strategies of many companies that, in turn, require new business models to be developed and new opportunities to work in the markets of new ideas and technologies to be provided.

We believe that it expedient for the Russian innovation system to undergo a research at the regional level using the concept of regional innovation systems (RIS). According to the model of network collaboration innovations (Gloor, 2006), innovations become interactive, and different social groups form certain innovation ecosystems become the sources of economic growth. That is, recently there has been taking place a transition from RIS-conceptions to the regional innovation ecosystem (Rothschild, 1990; Marshall et al., 1993) as a highly organized network of interaction between companies, embedded into a specific social and institutional context of a region. Such network is constantly changing. From this perspective the regional innovation system is a complex open system changing in time and containing complex feedback loops and response lags. A fundamental difference of the said conception from several others (national innovation system, learning region, industrial district, clusters, innovation environment) is a focus on such determining factors as network characteristics, trust between participants, sociocultural sets of the society, etc.

As for the interdisciplinary approach to analyzing innovation processes, it is covered by researchers in the field of regional digital ecosystems such as Habibipour A., Padyab A., Bergvall-Kåreborn B., Ståhlbröst A. (Habibipour et al., 2017); Ståhlbröst A., Bergvall-Kåreborn B. (Ståhlbröst and Bergvall-Kåreborn, 2013); Holst M., Stahlbrost A. (Bergvall-Kareborn, et al., 2009); Ley B., Ogonowski C., Mu M., Hess J., Race, N., Randall D., Rouncefield M., Wulf V. (Ley et al., 2015).

Building modern efficient regional innovation ecosystems requires to search and select methods, models and approaches to efficient management. Rapid development of digital technologies has made the ability to manage knowledge and to establish efficient interaction between participants of an innovation system the central aspects of management. Thus, open networks, open hardware, open data and open knowledge (European Union, 2015) have become the main trends of digital economy development.

In terms of the latest trends in digital economy development, we should consider the regional cyber-social system as a type of cyber-social systems (Sheth et al., 2013). Elementary cyber-social systems are systems of corporative, departmental and interdepartmental electronic workflow (Domrachev et al., 2016). 
The original term «cyber-social computing» is understood as the acquisition of demanded information from social networks and various cyber-physical systems of IoT (Internet of Things) in real time. Besides, this definition also fits special cloud infrastructures to support operations with data (Sheth, 2013). The cyber-social system is distinguished fundamentally by focusing on individuals (particularly, on their knowledge, abilities, sociocultural peculiarities) as an integral part of the system together with physical space (Liu et al., 2011).

The authors of the present article have been studying the neo-institutional approach to researching innovation systems represented in a number of works (Gamidullaeva, 2016a; 2016b; Gamidullaeva et al., 2017a) and suggest to consider the regional innovation system through the prism of institutions (formal and informal) and transaction costs. The goal of building an efficient innovation system according to the said approach is to reduce economic agents' transaction costs, which are the main barrier for innovations, especially if they are high (Gamidullaeva, 2016a; Gamidullaeva et al., 2017a; 2017b).

We shall generalize that under the regional innovation system in the modern conditions of digital transformation of the economy one should understand a complex open cyber-social system of the mesoeconomic level changing in real time and uniting economic agents, regional sociocultural factors (values and behavioral sets), formal and informal institutions at micro, meso and macrolevels.

We should point out that all elements of the regional cyber-social innovation system largely depend on safe and reliable access to key information systems to perform innumerable transactions within the chain of added value creation. Most processes in such a system may be accomplished without human involvement, as some systems in a region become strongly integrated, interconnected and interrelated. At the same time the networks not just transfer information, but with the help of the artificial intelligence elements embedded they are capable of accumulating this information, assessing it and even giving answers to questions and suggesting solutions using cutting-edge technologies of Data Mining and Data Analytics.

\section{Research Methodology}

Regional IS management is focused simultaneously on maintaining the system's stability and searching for new development options. The synergetic approach to management is based on the fact that there are multiple paths of IS development (scenarios with different probabilities), although it is necessary to reach the required attractor meeting the management goals. The identification of attractors in the field of predictability of regional innovation systems and their analysis make it possible to interpret dynamically the system's behavior with certain precision, which is especially important regarding the arrangement of "smart" interactions of innovation actors in the system. 
In this case it is also necessary to have well-organized feedback, as the system development is provided only by positive feedback in the system. By revealing the most important order parameters creating sorts of "fields of attraction" and subduing the behavior of all system elements, there rises an opportunity to develop a regional innovation system.

Thus, we suggest to use the synergetic approach as the methodological basis, according to which the system management should be performed by mechanisms of self-organization. At the same time, growing numbers of interactions between the system's elements should lead to increased organization and stability of the system. In modern conditions of digital transformation of the economy all inefficient interactions result in growing transaction costs that demotivate company owners to develop and implement innovations.

Consequently, it is necessary to create an innovation system, in which by following common goals of development the actors efficiently and intensively interact creating knew knowledge, exchanging it, apprehending and using it in the course of innovation activities. For this purpose, the actors require an intelligent medium for interaction. To form such medium the present research is to complete the following objectives:

$\checkmark$ to stratify innovation enterprises by regions;

$\checkmark$ to determine the order parameters of a regional innovation system to breakdown innovation enterprises into clusters by levels of the innovation activity and innovation susceptibility;

$\checkmark$ to breakdown enterprises into clusters determining the optimal number of clusters;

$\checkmark$ to identify attractors in each cluster featuring the best characteristics (parameters) of enterprises from the point of view of innovation activity and susceptibility;

$\checkmark$ to rate attractors in each stratum by the level of innovation activity and innovation susceptibility of enterprises;

$\checkmark$ to rate all enterprises in a cluster by the level of correspondence to attracting enterprises;

$\checkmark$ to determine indicators that influence the innovation activity and susceptibility of enterprises to the greatest extent using benchmarking and pairwise comparison;

$\checkmark$ to compile a list of enterprises with the greatest potential to be included into attracting enterprises;

$\checkmark$ to develop a map of innovation enterprises in a region grouped by the level of innovation activity and susceptibility with the purpose of building "smart" interactions of actors of innovation systems. 
In the course of the research, it is planned to develop models, methods and algorithms to solve problems of intelligent analysis and forecasting of the level of company and region's innovative development by means of the following:

$\checkmark$ synthesizing a complex of indicators and integral criteria of innovative development;

$\checkmark$ developing the optimal way (in the scope of project problem solving) of breaking down places of innovative development in Russian regions into strata on the basis of a synthesized complex of indicators for stratification;

$\checkmark$ searching for data on open-access Internet sources to calculate indicators of innovative development;

$\checkmark$ synthesizing models to estimate sensitivity and to select principal indicators (order parameters, clustering indication) of innovative development;

$\checkmark$ hierarchic multilevel clustering of economic subjects in the medium of selected indicators characterizing the degree of their innovative development in real time;

$\checkmark$ rating and selecting innovation leaders and "standards" for benchmarking;

$\checkmark$ comparative analyzing (benchmarking) of enterprises and innovation leaders to reveal factors and reasons for lagging behind investment leaders.

We shall give a closer look on study approaches to be used when solving such problems. The stratification method implies division of settlements, where innovation enterprises are located, into strata taking into account population sizes and regional features. As a result, there are obtained lists of settlements in strata. Stratification determines differences between strata and a set of indicators for optimal clustering of companies according to their location. Stratification requires various data including a list of operations aimed at acquiring the optimal number of strata by population sizes and other regional features to subsequently breakdown enterprises into clusters by a selected set of indicators with geo-spatial binding to the location of strata and companies in a stratum.

It is proposed to use 2 rules in accordance with mathematical statistics methods, such as dispersion and factor analysis, to assess and analyze possible ways of stratification. The first rule offers to identify similarities and differences of strata by the level of innovation potential. The second rule suggests to reveal similarities and differences of strata by the amount and indicators of investment appeal.

To solve the clustering problem it is required to search for innovation enterprises in strata and to include them into the universum for further extraction of required information and analysis. Under the universum one should understand a multitude of enterprises, the information on which is available in open sources. For information search and extraction it is offered to apply the multi-agent approach that has proved itself efficient in various problems. To solve the given problem the approach suggests to develop searching agents using open Internet sources to extract characteristics required for estimation of innovation potential and investment appeal 
of all companies in the universum, including geo-spatial information, denominations, data on innovation and intellectual property and other data required for calculation of indicators and forecasting the dynamics of their changes under altering influence factors. It is offered to design the agents in the form of special searching robots (crawlers) using the crawler creating library in Python Scrappy language. Below is a list of open sources for searching and selection of indicators:

$\checkmark$ Official statistics of the Federal State Statistics Service of Russia, the Ministry of Economic Development of Russia, the Ministry of Finances of Russia, the Ministry of Education and Science of Russia, published on the corresponding websites;

$\checkmark$ Websites of core ministries and agencies, internet-portals, core sites and other information resources associated with the innovation activity of the Russian Federation's regions;

$\checkmark$ Statistical proceedings of National Research University "Higher School of Economics" published on websites;

$\checkmark$ Results of sociological research and expert's surveys in Russian regions, published on websites of sociological research centers and on websites of regional administrative bodies;

$\checkmark$ Results of sociological studies of the "Georating" Public Opinion Foundation, published on its website etc.

As the information on certain objects may be located simultaneously in multiple sources, then the integration problem will be solved excepting redundant information and adding the missing one. It is suggested to use databases PosgreSQL with address clearing and geotagging to consolidate information on a certain enterprise from different sources. Geotagging requires adding of latitude and longitude coordinates, as well as missing data in companies' post addresses. The geo-spatial information is added using the technology of consecutive direct (transforming addresses to coordinates) and inverse (transforming coordinates to addresses) geo-coding engaging well-known services (Yandex Geocoder or Nominatium). The procedure should also include the backward check of geo-spatial information validity. The ultimate goal is to search for replicas of concurrent coordinate data and to remove redundant records. Important problems of the project research are searching and selection of required indicators. Such indicators are necessary for the following objectives:

$\checkmark$ primary evaluation of economic subjects' innovation activities;

$\checkmark$ separation of settlement strata with innovation-active enterprises;

$\checkmark$ clustering at different levels and industrial sectors;

$\checkmark$ selection of innovation leaders in clusters benchmarking.

Besides the indicators, here it is important to reveal external and internal factors that directly and indirectly influence company's innovative development. These indicators are introduced into the mathematical benchmarking model as coefficients 
to obtain forecasts of the innovation potential and to study the factors influencing integral indicators.

For analyzing purposes, it seems to be convenient to divide the methods, applied when estimating institutions, into two large classes: quantitative and qualitative methods. Such division of methods meets the system analysis requirements consisting in combining models and methods of formal and informal representations, which assists development of methodologies, selection of methods of gradual formalization of problem situation displaying and analyzing.

Following the neo-institutional methodological approach, we propose to group the indicators according to the institutional approach that includes the following formal and informal institutions of the innovation medium and groups of indicators for them:

$\checkmark$ The institution of standard and legal support of the innovation activity. A group of indicators reflecting completeness of standard and legal support of the innovation activity in a stratum and a region. A group of indicators of the law-enforcement mechanisms development in a stratum and a region. A group of indicators of the federal and regional legislation coordination in the field of innovation activities.

$\checkmark$ The institution of regional development. A group of indicators of financial security of innovation activities in a stratum and a region. A group of indicators reflecting the presence of skillful personnel for innovative development. A group of indicators reflecting the presence of resources for innovative development.

$\checkmark$ The institution of strategic planning of innovation activities. A group of indicators reflecting the presence of formalized elements of strategic innovation planning.

$\checkmark$ The institution of professional education and science. A group of indicators showing the effectiveness of professional education in a stratum and a region. A group of indicators of higher education quality in a stratum and a region. A group of indicators of financial security of research and development in a stratum and a region.

However, for the solution of the clustering problem such number of indicators is excessive. Thus, we shall decrease the dimensions of the clustering indicators space. For this purpose, it is necessary to pick a subset of significant indicators (order parameters) out of the whole set, which have the greatest impact on the synthesized models. The significance of individual indicators regarding the innovation potential assessment is determined by the method of multivariate correlation-regression analysis, which makes it possible to avoid subjective appraisals and to ensure credibility of subsequently calculated integral indicators. The insignificant indicators are excluded to deal with the problem of muticollinearity of factors and dimensionality reduction. 
To select the indicators it is necessary to establish the interconnection of sensitive clustering indicators with companies' investment appeal and opportunities to increase the innovation potential. The set of order parameters for enterprises is a list of investment appeal indicators that characterize the following:

$\checkmark$ companies' investment activity in regions;

$\checkmark$ potential investors' activity;

$\checkmark$ optimality of the existing innovation infrastructure in clusters.

To assess the indicators' sensitivity we suggest to use the method of variance analysis and to build a multivariate model of multifactorial variance analysis. The main point is to study the influence of the chosen factors on effective indicators of companies (innovation potential and investment appeal) in strata. The variance analysis is based on the analysis of deviations of units of the aggregate under consideration from the arithmetical mean. The dispersion appears to be the deviation metrics. Here we determine the deviations caused by the impact of a factor, and then compare them with the deviations created by random factors. As a result, the choice falls on the indicators with greater impact on the result, instead of the impact of random factors. It is these indicators that we shall consider as significant factors impacting the result and as order parameters.

It is proposed to develop an algorithm analyzing the institutional context and characteristics of regional innovation systems to increase credibility of the order parameters' selection results in the main complex project regarding selection of significant indicators. The algorithm's performance leads to appraisals of the formal and informal institutional context of a region and to selection of the most significant indicators of the innovation activity for subsequent calculation of the innovation potential's sensitivity towards the chosen indicators. As a result, one shall obtain a ranked list of indicators, out of which one will select a group of most sensitive indicators for further clustering to solve the problem of appraising the innovation potential and investment appeal.

Having completed the two methods the next step is to pick the intersecting sets of indicators, which in turn will serve as criteria for clustering. The obtained set of clustering indicators should characterize the following:

$\checkmark$ companies' innovation activities in strata;

$\checkmark$ optimality of an innovation infrastructure in a strata.

The clustering implies development of a method of hierarchical multilayered clustering of enterprises by chosen groups of indicators. It is offered to use the method of KMeans as the base algorithm for development of the new clustering method. The base method requires setting a number of clusters and a number of initializations of the iteration process of clustering (initial centroids). The number of initializations depends on the time given for clustering. 
As a result of the method's performance, each stratum will have multiple clusters of innovation-active enterprises. Particularly, each enterprise in a stratum and a region, in industrial sectors, in layers of small, medium and large enterprises (in conformity with federal laws) should be subject to clustering. Here, regional affiliation and geographical location (satellite coordinates) are taken into account. During clustering, it is of principal importance that objects in one cluster would be similar to each other in terms of the chosen indicators, but differ from objects in other clusters. Distances between joining enterprises are a criterion of making a decision on a number of clusters. The novelty of the developed method is in development of an integrated mechanism of clustering with subsequent selection of enterprises included in several clusters and establishment of the interconnection between values of sensitive indicators of clustering and innovative development parameters.

In the obtained clusters, it is necessary to evaluate the indicators and to distinguish groups of innovation leaders. For this purpose we propose to create ranked selections of companies, comparing them with the official statistics on the number of active companies of such type. Within the selections it is required to determine leading companies in terms of innovations (10 or 15\% of top companies in the ranked list of all enterprises in a stratum should be considered as leaders) that will subsequently become the base for picking and calculation of the indicators characterizing a model of the reference innovation enterprise. Sets of such indicators would differ in each stratum and each region, as they take into account local peculiarities of innovative development. Among innovation leaders, it is necessary to pick companies that also lead in clusters of various levels and layers. It is these leaders that will represent reference enterprises.

In the framework of benchmarking approach, the developed mathematical model is utilized for comparing all innovative enterprises in clusters and for identifying factors of innovation lagging from leaders. The company's innovative development is also subject to prognostic appraisal in case of exclusion of some factors reducing the innovation potential and investment appeal for certain enterprises.

It is offered to apply benchmarking for comparing the identified innovative development leaders in strata. In the context of the research work it is suggested to benchmark through determining and adapting examples of efficient innovative development of leading companies in the field of innovations in a region, a stratum and a settlement aimed at qualitative optimization of the innovation potential of other enterprises. Benchmarking models and methods enable to analyze advantages and disadvantages of leaders' innovative development in order to elaborate and implement an efficient strategy of company's innovative development in a region taking into account peculiarities of regional development and the influence of multiple factors under the effect of random market fluctuation.

It is suggested to benchmark the selected leaders by pairwise comparison of their innovation potential and investment appeal on the basis of the developed nonlinear 
model of coupled Van der Pol equations (oscillators), in which the coefficients of interaction between equations correspond to the parameters of innovation correspondence between companies under comparison. In case of comparing an innovation enterprise with the reference one, these coefficients allow to reveal and calculate current and forecasted divergence of innovative development indicators. The model should also take into account the impact of company's own innovation activity indicators and external factors on its investment appeal and competitiveness in relation to companies in the same line of business. The universal tool package Mathematica by Wolfram Research is applied to synthesize a benchmarking model and to simulate and forecast company's innovation activity indicators during benchmarking.

The software and knoware of the intelligent analysis of data and the comparative analysis of innovation enterprises are supposed to be implemented in the form of a platform intended for studying and appraisal of the regional innovation potential, as well as for forecasting the regional innovative development. It implies development of an architecture and structure of platform tools. The platform is a set of intelligent analysis tools, forecasting simulation of the dynamics of company's innovation interaction, sub-systems of searching, extraction, integration and storage of data from open sources. All components remain in the informational and technical interaction and provide automation of large data volumes processed in the MapReduce model of distributed data processing with application of cloud technologies. The problem is complicated by large volumes of data on many companies from various lines of business in regions to be extracted from open Internet sources, refined, preliminarily processed and integrated into cloud storage.

This is why it is required to develop and implement a data flow architecture for data integration. Such architecture implies establishment of distributed processing of data flows on multiple nodes. Each flow is a complete process of data treatment from extraction to uploading into a data storage intended for a single innovation company. The data flow architecture is effective when using the additive data model, where growing information volumes stored lead to addition of new data objects, but not to changing of already existing ones in the model.

For the information analytical platform to function it is required to develop an algorithm of applying decision-making tools in the process of company and region's innovative development management, which includes completion of the following objectives: analyzing a current situation in a regional innovation system, searching and collection of information on innovation activity indicators to evaluate the innovative potential on the basis of BigData technology, selection of competent pairs of innovation enterprises, simulation and forecasting of company's innovative development, selection and evaluation of factors impacting company's innovation, synthesizing scenarios of company's innovative development, forecasting simulation of innovation indicators' dynamics, analyzing simulation results, selection of value vectors of factors for the optimal innovative development strategy, selection of 
recommendations to achieve optimal values of innovation indicators for an individual company in a stratum and for a region in whole, synthesizing rules of recommendations selection, delivering recommendations to decision makers.

\section{Conclusion}

The main result of the research is the development of the method of complex forecasting evaluation of the innovation potential and investment appeal of companies to manage regional innovative development on the basis of the intelligent analysis of large data volumes by the method of cluster analysis, multivariate analysis, benchmark analysis, mathematical simulation, multi-agent approach and data flow technologies of data searching and collection, cloud storing of data processing results.

As a result, there has been developed a methodological approach including a set of models, methods and algorithms of searching, intelligent and comparative analysis of large volumes of data on companies in regions. This approach makes it possible to obtain reliable information on potential capabilities of regional innovation systems, as well as to elaborate real recommendations focused on innovative development of economic subjects and regions. The implementation of the research results in regional management will unite developers and users of the innovative development system, intensify their interaction, generate applied experience of joint projects and reduce transaction costs for economic subjects and potential investors.

The scientific novelty and significance of the research are determined by the development of new methods of intelligent analysis of large volumes of data for evaluation and forecasting of the innovation potential and investment appeal of enterprises and Russian regions. The practical value is defined by the fact that the invented models and methods will enable development of complex tools and agents of data flow collection and processing of information, analysis and evaluation of regional innovative development in the form of an information analytical platform. The universal nature of the developed models and methods allows to apply them for solving other problems, for example, analyzing company's competitiveness, trading potential and appeal, production potential etc.

\section{References:}

Baheti R., Gill, H. 2011. Cyber-physical systems: The impact of control technology, 12(C), 161-166.

Bergvall-Kareborn, B., Holst, M., Stahlbrost, A. 2009. Concept design with a living lab approach. In 42nd Hawaii International Conference on System Sciences, HICSS 2009, 1-10.

Chesbrough, H. 2003. Open Innovation: The New Imperative for Creating and Profiting from Technology. Harvard Business School Press, Boston, MA.

Domrachev, A.A., Evtushenko, S.N., Kupriyanovsky, V.P., Namiot, D.E. 2016. About 
innovative initiatives of the EEA member states in the field of building a global digital economy. International Journal of Open Information Technologies, vol. 4, no. 9.

European Comission. 2015. Growing a digital social innovation ecosystem for Europe DSI final report. European Union, https://ec.europa.eu/futurium/en/system/files/ged/50nesta-dsireport-growing_a_digital_social_innovation_ecosystem_for_europe.pdf

Gamidullaeva, L.A., Chernetsov, M.V., Vasin, S.M. and Taktarova, S.V. 2017. Enhancing Economic Growth through Innovation in Russia: Identifying Key Incentives for Innovation Proceedings of the 30th International Business Information Management Association Conference (IBIMA) 8-9 November, Madrid Spain, Vision 2020: Sustainable Economic development, Innovation Management, and Global Growth. Gamidullaeva, L.A., Tolstykh, T.O. 2017. Transaction Costs, Institutions and Regional Innovation Development: the Case of Russia. Proceedings of the 30th International Business Information Management Association Conference (IBIMA) 8-9 November, Madrid Spain, Vision 2020: Sustainable Economic development, Innovation Management, and Global Growth.

Gamidullaeva, L.A. 2016a. Institutions in the development of innovation systems. Journal of Economic Regulation, 7(1), 93-103.

Gamidullaeva, L.A. 2106b. On the construction of the concept of innovation management system. Economical Revival of Russia, 4(50), 74-84.

Gloor, P.A. 2006. Collaborative Innovation through Swarm Creativity. Swarm Creativity, 19-48, doi:10.1093/acprof:oso/9780195304121.003.0003.

Gloor, P.A. 2006. Collaborative Innovation through Swarm Creativity. Swarm Creativity, 19-48. doi:10.1093/acprof:oso/9780195304121.003.0003.

Habibipour, A., Padyab A., Bergvall-Kåreborn B., Ståhlbröst A. 2017. Exploring Factors Influencing Participant Drop-out Behavior in a Living Lab Environment. In Stigberg, S., Karlsen. J., Holone. H., Linnes. C. (eds) Nordic Contributions in IS Research, SCIS 2017.

Ley, B., Ogonowski, C., Mu, M., Hess, J., Race, N., Randall, D., Rouncefield, M., Wulf, V. 2105. At home with users: a comparative view of living labs. Interact Computing, 27, 21-35.

Liu, Z. 2011. Cyber-physical-social systems for command and control. IEEE Intelligent Systems, 26(4), 92-96.

Marshall, A. 1993. Principles of political economy. Moscow: Progress, 415 p.

Rothschild, M. 1990. Bionomics. Economy as Ecosystem. NY.: Henry Holt and Company Inc., $423 \mathrm{p}$.

Sheth, A., Anantharam, P., Henson, C. 2013. Physical-cyber-social computing: An early 21st century approach. IEEE Intelligent Systems, 28(1), 78-82.

Ståhlbröst, A., Bergvall-Kåreborn, B. 2013. Voluntary contributors in open innovation processes. In Eriksson-Lundström, J.S.Z., Wiberg, M., Hrastinski, S., Edenius, M., Ågerfalk, P.J. (eds.) Managing Open Innovation Technologies, 133-149, Springer, Berlin.

Vasin, S., Gamidullaeva, L. 2017a. Regional reproduction of innovations and development of network approach. Innovations, 4(13).

Vasin, S., Gamidullaeva, L. 2017b. Development of Russian innovation system management concept. Innovations, 5(223), 34-40. 